Cita bibliográfica: Beloki-Marañon, U. y Mosteiro-Pascual, A. (2017). Análisis del modelo de vulnerabilidad para la toma de decisiones en el ámbito de los cuidados de las personas mayores [Analysis of the vulnerability model for decision making related to the care of the elderly]. Alternativas. Cuadernos de Trabajo Social, $24,29-42$. https://doi.org/10.14198/ALTERN2017.24.02

\title{
ANÁLISIS DEL MODELO DE VULNERABILIDAD PARA LA TOMA DE DECISIONES EN EL ÁMBITO DE LOS CUIDADOS DE LAS PERSONAS MAYORES ${ }^{1}$
}

\section{ANALYSIS OF THE VULNERABILITY MODEL FOR DECISION MAKING RELATED TO ELDERLY CARE}

\author{
USUE BELOKI MARAÑÓN \\ Universidad de Deusto. \\ usue.beloki@deusto.es \\ Amaia Mosteiro Pascual \\ Universidad de Deusto. \\ amaia.mosteiro@deusto.es
}

\section{Resumen}

Este artículo analiza la validez de la vulnerabilidad como modelo para la toma de decisiones relativas a la situación personal y socio-familiar de la persona mayor necesitada de cuidados. La adecuación del modelo responde a la centralidad del elemento riesgo en la toma de decisiones, a la naturaleza del cuidado y al respeto de los principios que inspiran los derechos de las personas mayores vulnerables. El concepto de vulnerabilidad entronca con una nueva concepción en la que la vulnerabilidad se fundamenta, no sólo en la pérdida de la autonomía, sino en la susceptibilidad de quiebra de los derechos de la dignidad e integridad en la vida cotidiana; en consonancia con una concepción humana existencialmente diversa y vulnerable. La incorporación de este enfoque al Derecho de la capacidad de la persona mayor y regulación de los servicios sociales, exigirá su toma en consideración en los informes y diagnósticos de Trabajo Social.

Palabras clave: Vulnerabilidad, personas mayores, toma de decisiones, cuidados.

\section{Extended abstract}

\section{Introduction}

The analysis in this article focuses on the validity of vulnerability as a model for decision-making related to

\begin{abstract}
This article analyses the validity of vulnerability as a model for decision-making regarding the personal, family and social situation of the elderly in need of care. The adequacy of the model meets the requirements of risk as a core element in decision making and those coming from the nature of care and from the respect for the principles that inspire the rights of vulnerable older people. The concept of vulnerability connects with a new conception of vulnerability, which is not only based on the loss of autonomy, but also on the susceptibility of breaking the rights of dignity and integrity in daily life; in accordance with a new conception of humanity as existentially diverse and vulnerable. The inclusion of this approach into the law of the capacity of the elderly and into the regulation of social services will require taking the analysis of vulnerability into consideration in social work diagnosis and reports.
\end{abstract}

Key words: Vulnerability, the elderly, decision making, care.

personal and socio-family situations of elderly people who require care.

The idea of risk is the key element of analysis in the vulnerability approach, which has historically been applied in studies on poverty, natural disasters and,

Recibido: 11/04/2017

Aceptado: 27/07/2017

Publicado: 21/12/2017

1. Este artículo ha sido presentado como ponencia en el Grupo de Trabajo 9.2. "Cuidados en la sociedad del riesgo: vulnerabilidad, ciudadanía y justicia", del VI Congreso de la Red Española de Políticas Sociales, celebrado en Sevilla los días 16 y 17 de febrero de 2017. 
later, in the realm of gerontology. Furthermore, the risk concept forms the core of the decision-making theory.

\section{Methods}

The starting hypothesis is as follows: given the convergence of the risk concept in the vulnerability approach and in the field of decision-making, analysis of vulnerability in older people constitutes a correct model for decision-making related to their care. The vulnerability model also addresses the demands arising from the type of care in the home and observance of the inspiring principles of laws that protect the elderly.

The article, which is theoretical, identifies the elements of the vulnerability model in elderly care and analyses the vulnerability concept from the perspective of older people's rights.

\section{Findings and discussion}

a. Components of vulnerability in older people who require care.

The vulnerability which affects the elderly (consisting of threats, exposure, possibility and extent of harm and confrontation) and the possibility of taking action in this area (Schröder-Butterfil \& Marianti, 2006; Schröder-Butterfill, 2013), are determined by their physical and mental health, cognitive capacity, social networks and support, material resources, housing, knowledge and experience, availability of social services as well as their individual personality, expectations and personal motivation. The following vulnerability conditions are important: declining health -including memory impairment-, limited mobility and capacity to carry out activities, pain and insecurity (Feito Grande, 2007; Delgado Rodríguez, 2012). Other key vulnerability conditions include a lasting internalised feeling of frailness, helplessness and personal insecurity (Filgueira, 2001; Busso, 2001; Cardona, 2001, Castel, 2014), associated with the risk concept and the perception of not being able to control their own destiny.

In vulnerable older people, this psychological condition commonly underlies the idea of vulnerability itself (Clement $\&$ Bolduc, 2004) that arises not only from frailness or dependence but also due to stressful events. These may be one-off or unexpected occurrences like the loss of the partner, falls and household accidents or hospitalization. Vulnerable older people are more exposed to critical events, which, together with greater frailness and dependence, hinder resilience in traumatic situations, given that such moments increase their mistrust and insecurity concerning the future (Ipsos Mori, 2014).

As for coping mechanisms, care in the home is characterised by informal and flexible strategies that are constantly adapted, with a strong relational component. This is consistent with the vulnerability felt by older people when facing difficulties.

\section{b) Vulnerability as overcoming lack of autonomy}

The vulnerability concept thus fits in a conception of the need for care that goes beyond the liberal idea of autonomy and is linked to man's anthropological vulnerability in which specific conditions of frailness and helplessness are also recognised (Ricoeur, 2008-a; Delgado Rodríguez, 2012; Martín Palomo, 2016).

The vulnerability concept is connected to a new idea in which difficulties are not only based on one's lack of autonomy but on how easily the right to dignity and integrity in daily life can be violated. This is in line with an existentially diverse and vulnerable view of man (Rendtorff, 2002; Palacios Rizzo, 2008; Gordillo Álvarez-Valdés, 2008; Requena Meana, 2008; Guzmán Castillo, Toboso Martín, \& Romañach Cabrero, 2010; Bermejo, 2014).

\section{c. Decisions related to naturalistic and shared types of care}

Decisions on care during ageing are characterised by having a direct impact on older people's lives, including emotional and evaluative elements and adopting to the daily socio-family context (Agrela Romero, Martín Palomo, \& Langa Rosado, 2010; Delgado Rodríguez, 2012; Ramos Ponzón, 2012; Bosch Fontcuberta, 2012). They refer to aspects which are familiar, understandable and manageable for older people (Salthouse, 1996; Wood \& Tanius, 2008; Foster et al., 2008; Craig \& Higgs, 2012). These decisions should therefore be based on the naturalistic theory (Klein, 1999) (Zsambok \& Klein, 1997) and shared mind (Epstein, 2013; Elwyn, 2012).

In contrast to a contractual relationship model which centres on informed consent and seeks to avoid intrusion in older people's lives and rights, shared naturalistic-based decisions are made with a focus on person-centred helping relationships. This is a constant negotiation process which includes reflection on the tasks and risks of daily personal and home life. Control and independence-related aspects are discussed and agreed on. Thus, a reflection of the logic that older people apply in their decisions on risks and difficulties is formed, based on normalisation and continuous adaptation strategies (Craig \& Higgs, 2012; Taylor $\&$ McKeown, 2013). In this manner, the elderly's empowerment and satisfaction are fostered while also promoting the co-responsibility of those taking part in the decision-making (Ipsos Mori, 2014).

\section{Conclusions}

Assessment of decision-making capacity in legislation which affects the elderly, as well as that which regulates social services in Spain, continues to be based on a rational idea of autonomy, which is the traditional vision found in Civil Law. In contrast to other regulations in which the vulnerability focus underpins the legal system which protects the elderly, the Spanish legal system continues to address the subject's individual autonomy and rational capacity for informed consent.

It would be advisable that the reform on capacity (Law/26/2015 of 28 July, modification of the system to protect children and adolescents) prompt changes in the determination of the elderly's legal decision-making capacity and go beyond a strictly cognitive vision to centre on assessing decision-making capacity related to daily care as persons understanding their vulnerability; in other words, a focus on older persons' capacity and will to overcome problems or defend themselves from threats in the environment as well as the existence or availability of the means to face them. These aspects should form part of the social diagnoses and reports issued from the discipline of Social Work with this aim in mind. Shared decision-making should also be considered if older people, regardless of their cognitive impairments, are to exercise their right to take part in decisions that affect them.

Key words: Vulnerability, the elderly, decision making, care, Social Work. 


\section{Introducción}

La vulnerabilidad en el ámbito de la gerontología se aborda prioritariamente desde dos enfoques: un primer enfoque asociado los derechos humanos de las personas mayores como grupo que en reiteradas ocasiones es objeto de una desigualdad material y de una discriminación indirecta; y un segundo enfoque ligado a la idea del riesgo, que considera a las personas mayores como un colectivo necesitado de especial cuidado y protección por su mayor susceptibilidad a sufrir un daño que resulta de diversas amenazas (Barranco Avilés, 2014).

Este segundo enfoque encuentra sus raíces en el concepto y modelos de la vulnerabilidad frente a la pobreza (Chambers, 1989; 1995) y ante los desastres naturales (Blaikie et al., 1994). Su desarrollo posterior persigue capturar la diversidad y la complejidad de los riesgos contemporáneos junto con la variedad de formas de desprotección, inseguridad e incertidumbre (Busso, 2002). Hoy cabe afirmar que los grupos vulnerables pueden ser tantos como riesgos a los que están expuestos (Sánchez González et al., 2012) y su análisis permite prevenir o minimizar los daños a través de la toma de las decisiones más adecuadas.

El enfoque de la vulnerabilidad se aplica en la gerontología posterior a los noventa (Tullman \& Chang, 1999; Schröder-Butterfil \& Marianti, 2006; Andrew et al., 2008; Bilotta et al., 2010; Brodiez-Dolino, 2013; 2014), bien para analizar las condiciones sociales de un grupo que se enfrenta a los riesgos derivados del envejecimiento, bien desde el nivel micro o meso-social de la persona mayor, como sujeto que vive su vulnerabilidad de forma personal y circunstancial (Brodiez-Dolino, 2014; 2016; Clement \& Bolduc, 2004; Hall, 2014; Naik et al., 2010;). Afirma en este sentido Martín Palomo (2016: 167) que se impone «analizar el cuidado y las vulnerabilidades desde perspectivas micro, para conocer cómo las formas diversas de vulnerabilidad se manifiestan en hombres y mujeres concretos que tienen sus problemas concretos».

\section{El concepto de vulnerabilidad y sus componentes en el colectivo de personas mayores}

La vulnerabilidad en el segundo de los enfoques citado se define por la exposición de una persona, familia, grupo o comunidad a uno o varios riesgos determinados, así como por la previsible incapacidad de respuesta para hacer frente a los mismos (CEPAL-CELADE, 2002). En síntesis, la vulnerabilidad hace referencia a la factibilidad de que un sujeto, grupo o comunidad se vea afectado por el daño que se deriva de una amenaza (Cardona, 2001). La propia exposición a la amenaza, la incidencia del daño, así como la capacidad de 
anticipación o de respuesta están determinadas por los condicionantes estructurales, sociales y personales (Bello Sánchez \& González Rego, 2012). De esta forma, la vulnerabilidad de quien está en ella se presenta como un proceso multidimensional en el que confluyen todos los elementos -factores internos y externos-, que dan lugar al riesgo o a la probabilidad del daño en un tiempo y un espacio determinado (Busso, 2001). Como señala Katzman (2000: 281), «las situaciones de vulnerabilidad suelen desencadenar sinergias negativas que tienden a un agravamiento progresivo».

Este carácter multidimensional y acumulativo exige tener en cuenta no sólo los componentes de la vulnerabilidad (amenaza, grado de exposición, posibilidad de generación de un daño, magnitud del mismo y capacidad de afrontamiento), sino también las relaciones que se establecen entre ellos. La virtualidad del enfoque de la vulnerabilidad radica, precisamente, en la posibilidad de actuar en cada uno de esos niveles: sobre la propia existencia de la amenaza, sobre la exposición de la persona, hogar o comunidad, sobre su capacidad de afrontamiento y sobre la eventualidad del daño (Schröder-Butterfil \& Marianti, 2006; Schröder-Butterfill, 2013).

Figura 1. Un marco para comprender la vulnerabilidad

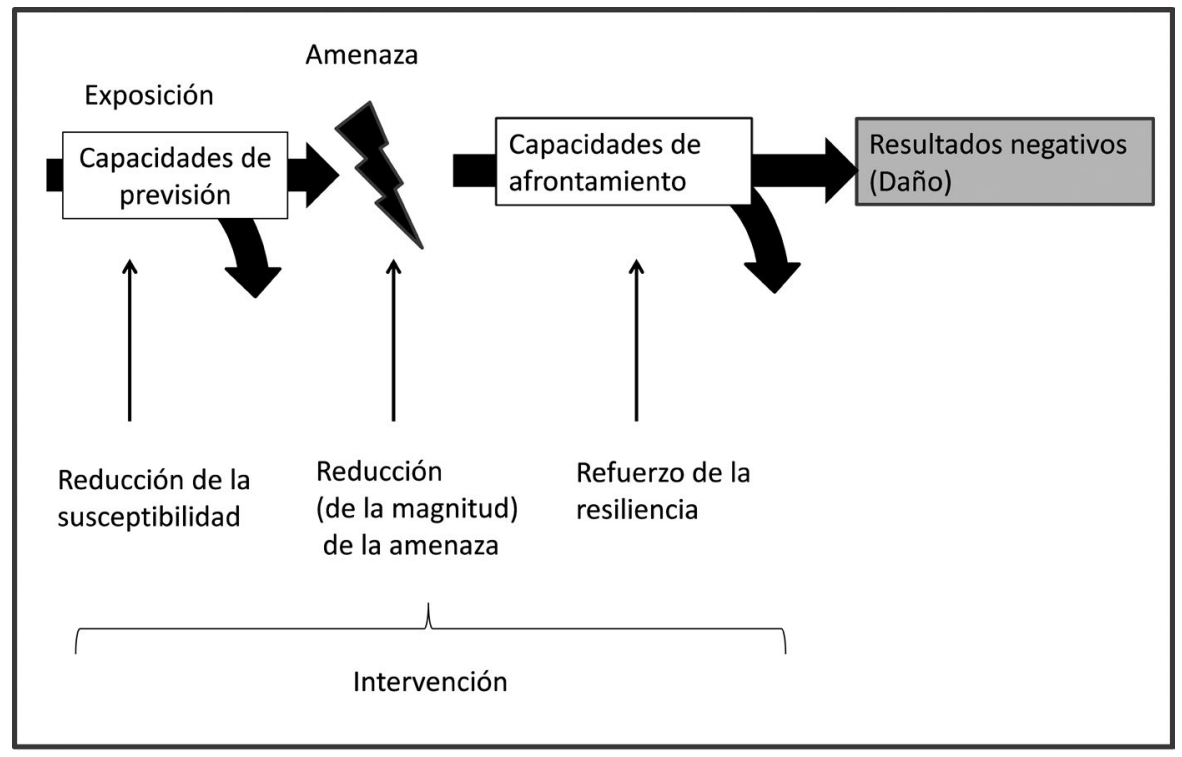

Fuente: Adaptado de Schröder-Butterfill (2013) 
La vulnerabilidad adquiere distintas formas: así, la debilidad interna o fragilidad del individuo, su indefensión ante el entorno, el desamparo institucional o la incapacidad e inseguridad a la hora de aprovechar oportunidades y pensar estrategias para la mejora del bienestar (Busso, 2001). En el caso de las personas mayores su vulnerabilidad depende de un amplio espectro de factores de salud física, mental, capacidades cognitivas, redes y apoyo social, recursos materiales, vivienda, conocimiento y experiencia, disponibilidad de servicios sociales, personalidad individual y las expectativas y motivaciones personales. Entre ellos se apuntan como condiciones de vulnerabilidad más destacables las siguientes: el declive de la salud -incluido el deterioro de la memoria-, las limitaciones de la movilidad y de la capacidad de realización de actividades, el dolor, y la inseguridad (Feito Grande, 2007; Delgado Rodríguez, 2012).

El enfoque incorpora la vertiente subjetiva de la vulnerabilidad, es decir, el sentimiento de inseguridad que resulta de las condiciones de la vejez y que tiene un fuerte impacto en el bienestar percibido de la persona mayor. La vulnerabilidad va más allá de la percepción puntual de las amenazas y se convierte en un sentimiento permanente en la vida de la persona mayor, vinculado a sensaciones de fragilidad, indefensión e inseguridad personal que se mantienen en el tiempo (Filgueira, 2001; Busso, 2001; Castel, 2014), y que pueden llegar incluso a afectar a la identidad personal (Cardona, 2001). Este sentimiento surge de la incertidumbre asociada al propio concepto de riesgo (Clement \& Bolduc, 2004) - de la indeterminación de que la amenaza finalmente tenga lugar o de que el daño se produzca-, así como de la percepción de la eventual incapacidad de controlar el propio destino. En el caso de las personas mayores vulnerables, a este estado psicológico subyacente a la noción misma de vulnerabilidad se añade el que resulta del impacto de acontecimientos estresantes imprevistos, tales como la pérdida de personas significativas, las caídas y accidentes domésticos, y los ingresos hospitalarios. La persona mayor vulnerable está más expuesta a acontecimientos críticos, tiene menos reservas físicas (fragilidad) y funcionales (dependencia) para responder a una crisis o trauma (resiliencia), y éstos constituyen habitualmente puntos de inflexión en relación con la confianza y seguridad en la situación personal futura (Ipsos Mori, 2014).

\section{Los activos relacionales de las personas mayores para hacer frente a la vulnerabilidad}

El estudio de la vulnerabilidad incluye el análisis del manejo de los recursos y de las estrategias que utilizan las comunidades, familias y personas para enfrentar los efectos del riesgo (Pizarro, 2001). Los colectivos vulnerables ponen en 
marcha sus propias estrategias en función de su situación particular y de sus convicciones. El grupo de personas mayores afronta los riesgos derivados de la vejez recurriendo a los activos personales, a estrategias familiares y comunitarias de cuidado informal, así como a los servicios organizados públicos o privados. Señala Moreno en referencia a los activos informales que, aunque parte de ellos dependan muchas veces del voluntarismo y sean fuente en último término de desigualdad, se caracterizan por una inmediatez y flexibilidad que constituye un gran alivio para quien los utiliza (Moreno, 2001).

El enfoque pone así de relieve el carácter relacional del afrontamiento de las dificultades durante el envejecimiento. Ello supone la superación de una concepción liberal del concepto de autonomía, que había centrado su atención en la garantía de la libertad, independencia y espacio vital libre de injerencias externas (Delgado Rodríguez, 2012) que al mismo tiempo, sin embargo, había permanecido ignorante de la influencia del contexto en la situación de vulnerabilidad.

El modelo de la vulnerabilidad parte de una vulnerabilidad antropológica - «entendida como una condición de fragilidad propia e intrínseca al ser humano, por su ser biológico y psíquico»-, que puede verse modulada por las circunstancias personales o por el propio contexto o dimensión social, que repercuten en la susceptibilidad al daño y en la consecución de la mayor autonomía y calidad de vida (Feito Grande, 2007: 8). Aunque el enfoque admite que el ser humano es esencialmente vulnerable e interdependiente, reconoce que existen personas con mayor fragilidad, indefensión y vulnerabilidad (Martín Palomo, 2016). Se incorpora así el concepto de autonomía relacional como medio para alcanzar la autonomía personal (Ricoeur, 2008-a; Delgado Rodríguez, 2012), meta que además se comparte con otros principios como la dignidad o la integridad (Gordillo Álvarez-Valdés, 2008; Rendtorff, 2002; Requena Meana, 2008), en consonancia con la concepción del vulnerable como la de una persona con «otra manera de ser en el mundo» (Ricoeur, 2008-b: 181). La dignidad deja de estar concebida como un atributo consustancial a la condición de autonomía y racionalidad, como un derecho humano elaborado sobre la capacidad de razonar, sentir o comunicar y se predica de la persona que «es» (Bermejo, 2014; Guzmán Castillo, Toboso Martín, \& Romañach Cabrero, 2010; Palacios Rizzo, 2008).

\section{Vulnerabilidad, toma de decisiones compartida y cuidados cotidianos}

En el enfoque de la vulnerabilidad la persona mayor necesitada de cuidados deja de ser objeto de clasificaciones dicotómicas o categorizadas de capacidad o incapacidad, o de autonomía o dependencia, y pasa a ser considerada como 
una persona con unas condiciones y en un contexto que le hace vulnerable a una disminución de su bienestar. Esta concepción se traslada en términos prácticos al ámbito de la toma de decisiones relativas a la persona mayor, en las que ocupan un lugar central las relativas al cuidado.

Las decisiones sobre los cuidados durante el envejecimiento tienen un impacto directo en la vida de la persona mayor, incorporan elementos emocionales, valorativos y se refieren a aspectos que le resultan cercanos, comprensibles y en cierto sentido manejables (Salthouse, 1996; Wood \& Tanius, 2008; Foster et al., 2008; Craig \& Higgs, 2012). Bajo este prisma resulta coherente que las decisiones sobre el cuidado -en el sentido de caring about y de caring for (Tronto, 1998) -, se tomen sobre la base de la teoría naturalista de la toma de decisiones (Zsambok \& Klein, 1997) y como parte de un proceso interactivo y compartido entre los distintos agentes implicados (shared mind) (Epstein, 2013; Elwyn, 2012). Las decisiones relativas a los cuidados incorporan componentes emocionales, intuiciones o elementos críticos (whole mind) que junto con el conocimiento profesional forman parte del conocimiento compartido (Levin, 2012; Cardona-Cardona et al., 2017). Por lo que respecta al proceso, se trata de un proceso de comunicación y negociación constante en el que afloran los valores personales, se reflexiona sobre las tareas y riesgos de la vida personal y doméstica cotidiana y se discuten y acuerdan aspectos relativos al control y a la independencia. Ello no es sino un reflejo de la lógica que siguen las personas mayores en sus decisiones sobre los riesgos y dificultades, que está basada en la normalización y en estrategias de adaptación continuas (Craig \& Higgs, 2012; Taylor \& McKeown, 2013).

Frente a un modelo de relación contractual, que tiene como elemento central el consentimiento informado y que persigue la no intromisión en la vida y derechos de la persona mayor, las decisiones compartidas se toman bajo el enfoque de la relación de ayuda centrada en la persona (Delgado Rodríguez, 2012). Se reconoce la autonomía relacional de la persona mayor y se promueve su autogobierno, partiendo de su interdependencia del contexto socio-familiar cotidiano en el afrontamiento de las condiciones de vulnerabilidad (Agrela Romero, Martín Palomo, \& Langa Rosado, 2010) y tomando en consideración el hecho de que las personas significativas son aquellas con las que habitualmente se consultan y reflexionan las decisiones relevantes. (Delgado Rodríguez, 2012; Ramos Ponzón, 2012; Bosch Fontcuberta, 2012).

En un plano práctico, la toma de decisiones compartida permite decidir con la persona mayor vulnerable que lo autorice, la continuidad de su participación en las tareas cotidianas que impliquen un riesgo, o la incorporación, en su caso, de los cambios, ajustes y adaptaciones necesarias. Se trata de elevar las 
expectativas de lo que es posible a los niveles más altos, con el fin de promover el empoderamiento y la satisfacción de la persona mayor, al tiempo que se favorece la corresponsabilidad de quienes participan en la toma de decisiones (Ipsos Mori, 2014).

\section{Discusión: la aplicabilidad del modelo a la toma de decisiones relativas a personas mayores vulnerables con dificultades cognitivas}

La toma de decisiones compartida puede ponerse en cuestión cuando la persona mayor vulnerable tiene sus capacidades cognitivas mermadas y su participación en el proceso de toma de decisiones queda por ello cuestionado ab initio. La consideración de las opiniones, emociones y deseos de esta persona y la participación de familiares o personas allegadas tiene cabida en el principio deontológico de trabajo social de promoción integral de la persona, pero su encaje entraña una mayor dificultad en la regulación actual de los servicios sociales. Cabe afirmar que la normativa de los servicios sociales descansa todavía sobre un concepto racional de la autonomía, de corte cognitivo, que subraya el consentimiento informado propio del Derecho civil tradicional. A diferencia de lo que sucede en el ámbito anglosajón, en el que el enfoque de la vulnerabilidad subyace en el sistema legal protector de las personas mayores (ABA, 2005; 2006; Care Act de 2014 del Reino Unido, secciones 42-7) -posibilidad que es objeto de análisis también en los últimos años en el Derecho civil Francés (Rebourg \& Bourdin, 2014)-, en el ordenamiento jurídico español, permanece todavía un enfoque basado en la autonomía individual y en el consentimiento. La normativa autonómica reguladora de los servicios sociales que se aprueba en la primera década del nuevo milenio incorpora expresamente en su articulado como principio regulador la autonomía de las personas usuarias sobre su propio proyecto vital, así como su derecho a consentir, a renunciar o a adoptar decisiones libres sobre las intervenciones sociales, supeditadas a la legislación civil vigente, en aquellos casos de personas incapacitadas o presuntas incapaces.

La recepción del Convenio sobre los Derechos humanos de las personas con discapacidad, de 13 de diciembre de 2006, operada en el ordenamiento jurídico civil con las últimas reformas relativas a la modificación de la capacidad (Ley 26/2015, de 28 de julio, de modificación del sistema de protección de la infancia y de la adolescencia), sienta las bases para un cambio en la normativa de servicios sociales que supere la tradicional dicotomía entre capacidad e incapacidad. La instauración del sistema de apoyos necesarios para la promoción de la toma de decisiones de la persona con discapacidad, -incluidas las personas mayores-, y la creciente diferenciación de las esferas de la persona a los efectos 
de la valoración de su capacidad, permiten vaticinar un área de desarrollo del trabajo social. La consideración de las habilidades para la vida independiente como área específica de valoración en los procesos de modificación de la capacidad (Instrucción n. ${ }^{\circ}$ 3/2010 de la Fiscalía General del Estado) ubicará al trabajador/a social como un agente clave en la determinación de la capacidad de la persona mayor vulnerable con deterioro cognitivo (Rueda, 2012), así como en la promoción de su autonomía, también en sentido relacional. Procede en estos casos entonces reconstruir lo que fuera su voluntad, preferencias y valores conciliándolos, en la medida de lo posible, con la gestión del riesgo que en el momento actual configura su vulnerabilidad.

\section{Conclusiones}

Como se ha dicho hasta el momento, los cuidados de las personas mayores vulnerables se caracterizan por la confluencia de aspectos sociales y sanitarios, con implicaciones socio-familiares, en los que está presente un estado psicológico de incertidumbre y cuya resolución actual depende de la puesta en marcha de activos, en gran medida informales. Es lugar común que el trabajo social tiene como uno de sus elementos distintivos la libertad e individualidad consideradas en el contexto social de la persona. El respeto de la voluntad libremente formada de la persona mayor y la promoción de su red de apoyo, con la toma en consideración de sus opiniones, forman parte de los principios y fundamentos de la disciplina.

Si el modelo de la vulnerabilidad resulta de utilidad para que profesionales, personas mayores y personas significativas identifiquen el riesgo y los activos disponibles y establezcan estrategias conjuntas para su gestión; cuando la persona mayor tiene sus facultades cognitivas mermadas las decisiones relativas a sus cuidados exigen, además, un trabajo social dirigido a identificar y analizar los elementos sociales que permitan valorar la capacidad de esta persona de comprender, asumir y gestionar su propia vulnerabilidad.

Los riesgos que resultan del contexto, la necesidad de recepción de cuidados y la comprensión de la persona mayor de su propia situación constituyen elementos valorativos que habrán de formar parte de los diagnósticos e informes sociales que a tal fin se emiten desde la disciplina del trabajo social. Asimismo, la toma de decisiones compartida habrá de ser considerada si se quiere hacer efectivo el derecho de la persona mayor, -aun con dificultades cognitivas- a participar en las decisiones que le afectan. 


\section{Bibliografía}

ABA. American Bar Association. Commision on Law and Aging \& American Psichological Association. (2005). Assessment of Older Adults with Diminished Capacity: A Handbook for Lawyers. Washington, DC: ABA. American Bar Association \& American Psichological Association. Disponible en: http://www. apa.org/pi/aging/resources/guides/diminished-capacity.pdf

ABA. American Bar Association. Commision on Law and Aging \& American Psichological Association. (2006). Judicial Determination of Capacity of Older Adults in Guardianship Proceedings. Handbook for Judges. Washington DC: American Bar Association \& American Psychological Association. Disponible en: https://www.apa.org/pi/aging/resources/guides/judges-diminished.pdf

Agrela-Romero, B., Martín-Palomo, M. T. y Langa-Rosado, D. (2010). Modelos de provisión de cuidados: género, familias y migraciones. Nuevos retos y configuraciones para las políticas. Alternativas. Cuadernos de Trabajo Social, 17, 9-17. https://doi.org/10.14198/ALTERN2010.17.1

Andrew, M., MitnitsK, A. \& RocKWOOD, K. (2008). Social Vulnerability, Frailty and Mortality in Elderly People. PLoS ONE, 3(5). https://doi.org/10.1371/ journal.pone.0002232

BARRANCO AVILÉs, M. (2014). Derechos humanos y vulnerabilidad. Los ejemplos del sexismo y del edadismo. En M. Barranco Avilés y C. Churruca Muguruza, Vulnerabilidad y protección de los derechos humanos (16-44). Valencia: Tirant lo Blanch.

Bello SÁnChez, W. y González Rego, R. A. (2012). Vulnerabilidad de las personas de la tercera edad ante el riesgo de envejecimiento en Cuba. En C. Ejea Jiménez, D. Sánchez González y J. I. Soledad Suescún, Vulnerabilidad Social. Posicionamientos y ángulos desde geografías diferentes (201-215). Granada: Eirene.

BERMEJO, L. (2014). Estudio sobre la creación de una unidad de calidad de vida en personas con demencia en sus fases iniciales. Madrid: Ministerio de Sanidad y Servicios Sociales e Igualdad. Disponible en: http://www.fundacionreinasofia. es/Lists/Documentacion/Attachments/66/12015_unidad_calidad_vida.pdf

Bilotta, C., Casè, A., Nicolini, P., Mauri, S., Castelli, M. \& Vergani, C. (2010). Social vulnerability, mental health and correlates of frailty in older outpatients living alone in the community in Italy. Ageing and Mental Health, 14(8), 10271036. https://doi.org/10.1080/13607863.2010.508772

Blaikie, P., Cannon, T., Davis, I. \& Wisner, B. (1994). At Risk: Natural Hazards, People Vulnerability and Disasters (1st. ed.). London-New York: Routledge.

BosCh FontCUBERTA, J. M. (2012). La toma de decisiones conjunta en medicina: una difícil asignatura. Revista de atención primaria, 17, 385-387. https://doi. org/10.1016/j.aprim.2012.05.010 
Brodiez-Dolino, A. (2013). Combattre la pauvreté. Vulnérabilités sociales et sanitaries de 1880 à nos jours. Paris: CNRS.

Brodiez-Dolino, A. (2014). La vulnérabilité, entre sanitarie et social. En A. Brodiez-Dolino, I. Von Vueltzingsloewen, B. Eyraud, C. Laval, \& B. Ravon, Vulnérabilités sanitaires et sociales (9-16). Rennes Cedex: Presses Universitaires de Rennes.

Brodiez-Dolino, A. (2016). Le concept de vulnérabilité. Disponible en: www.laviedesidees.fr.

Busso, G. (2001). Vulnerabilidad social: nociones e implicancias de políticas para latinoamérica a inicios del siglo XXI. Seminario Internacional «Las Diferentes expresiones de la Vulnerabilidad Social en América Latina y el Caribe», 20 y 21 de junio. Santiago de Chile: Naciones Unidas. Comisión Económica para América Latina y el Caribe. CEAPAL. Disponible en: http://www.cepal.org/ publicaciones/xml/3/8283/GBusso.pdf

Cardona-Cardona, J. Cuartero-Castañer, M. E. y Campos-Vidal, J.F. (2017). La comprensión de la situación problema en la práctica del Trabajo Social de Casos. Resultados de una investigación. Cuadernos de Trabajo Social, 30(1) 2017: 149-162. http://dx.doi.org/10.5209/CUTS.51735

CARDONA, O. D. (2001). La necesidad de repensar de manera holística los conceptos de vulnerabilidad y riesgo: una crítica y una revisión necesaria para la gestión. Memoria International Work-Conference on Vulnerability in Disaster Theory and Practice. Disponible en: http://www.desenredando.org/public/ articulos/2001/repvuln/RepensarVulnerabilidadyRiesgo-1.0.0.pdf

CASTEL, R. (2014). Los riesgos de exclusión social en un contexto de incertidumbre. Revista Internacional de Sociología (RIS), 72 (Extra 1), 15-24. Procesos de exclusión social en un contexto de incertidumbre). https://doi.org/10.3989/ ris.2013.03.18

CEPAL-CELADE. (2002). Vulnerabilidad sociodemográfica: viejos y nuevos riesgos para comunidades, hogares y personas. Brasilia: CEPAL-CELADE. Naciones Unidas. Disponible en: http://www.cepal.org/publicaciones/xml/4/11674/ LCW3-Vulnerabilidad.pdf

Chambers, R. (1989). Vulnerability, Coping and Policy. IDS Bulletin, 20(2), 1-7. https://doi.org/10.1111/j.1759-5436.1989.mp20002001.x

CHAMBERS, R. (1995). Poverty and livelihoods: whose really counts? Environment and Urbanization, 7(1), 173-204. http://www.archidev.org/IMG/pdf/p173.pdf

Clement, M. \& Bolduc, N. (2004). Regards croisés sur la vulnérabilité. En F. Saillant, M. Clément \& C. Gaucher, Identités, vulnérabilités, communautés (6182). Montréal: Les Éditions Nota Bene.

Craig, G. M. \& HigGS, P. (2012). Risk owners and risk managers: Dealing with the complexity of feeding children with neurodevelopmental disability. 
Negotiating and communicating health risk. Health, Risk \& Society, 14 (7-8), 627-637. https://doi.org/10.1080/13698575.2012.717610

DELGADO RodríGUEZ, J. (2012). Nuevas perspectivas bioéticas: autonomía relacional. Revista de Enfermería, 6 (1), 35-42. Disponible en: http://ene-enfermeria. org/ojs/index.php/ENE/article/view/8/7

Elwyn, G., Frosch, D., Thomson, R., Joseph-Williams, N., Lloyd, A., Kinnersley, P. \& BARry, M. (2012). Shared Decision Making: A Model for Clinical Practice. Journal of General Internal Medicine, 27(10), 1361-1367. https://doi. org/10.1007/s11606-012-2077-6

EPSTEIN, R. M. (2013). Whole mind and shared mind in clinical decision-making. Patient Education and Counseling, 90, 200-206. https://doi.org/10.1016/j. pec.2012.06.035

España. Ley 26/2015, de 28 de julio, de modificación del sistema de protección a la infancia y a la adolescencia. BOE núm. 180, de 29 de julio de 2015. 64544-63613

Feito Grande, L. (2007). Vulnerabilidad. Anales del Sistema Sanitario de Navarra, 30 (3), 7-22. Disponible en https://recyt.fecyt.es/index.php/ASSN/article/ view/2004/1431

FILGUEIRA, C. H. (2001). Estructura de oportunidades y vulnerablidad social aproximaciones conceptuales recientes. Santiago de Chile: CEPAL. Disponible en: http://www.cepal.org/publicaciones/xml/3/8283/cfilgueira.pdf

Fiscalía General del Estado. Instrucción n. ${ }^{\circ}$ 3/2010 sobre la necesaria fundamentación individualizada de las medidas de protección o apoyo en los procedimientos sobre determinación de la capacidad de las personas. http://www.elsindic.com/documentos/586_INSTRUCCION\%203-2010\%20FISCALIA\%20GENERAL\%20 DEL\%20ESTADO.pdf

Foster, S., Cornwell, R., Michael, A. \& Davis, H. (2008). Cognitive Changes across the Life Span. En S. H. (Ed.), Changes in Decision-Making Capacity in Older Adults. Assesment and Intervention. New Jersey: John Wiley \& Sons, Inc.

GORDILlO ÁlVAREZ-VALDÉs, L. (2008). ¿La autonomía, fundamento de la dignidad humana? Cuadernos de Bioética (66), 237-253.

Guzmán Castillo, F., Toboso martín, M. y Romañach Cabrero, J. (2010). Fundamentos éticos para la promoción de la autonomía: hacia una ética de la interdependencia. Alternativas. Cuadernos de Trabajo Social, 17, 45-61. https:// doi.org/10.14198/ALTERN2010.17.03

Hall, M. (2014). Dementia, Autonomy and Guardianship for the Old. En J. H. Charles Foster, The Law and Ethics of Dementia (339-349). Oxford: Hart Publishing.

IPSOS MORI. (2014). Understanding the lives of older people living with frailty. A qualitative investigation. London: Age UK. Disponible en: https://www. ipsos-mori.com/DownloadPublication/1660_sri-ipsos-mori-age-uk-livingwith-frailty-2014.pdf 
KATZMAN, R. (2000). Notas sobre la mediacion de la vulnerabilidad social. Santiago de Chile: CEPAL. Disponible en: http://www.cepal.org/deype/mecovi/docs/ taller5/24.pdf

LEVIN, L. (2012). Towards a revised definition of client collaboration: the knowledge-power-politics triad. Journal of Social Work Practice, 26 (2), 181195. https://doi.org/10.1080/02650533.2010.545121

Martín PAlOMO, M. T. (2016). Cuidado, vulnerabilidad e interdependencias. Madrid: Centro de Estudios Políticos y Constitucionales.

Moreno, L. (2001). Estados del bienestar y «mallas de seguridad». En L. Moreno, Pobreza y exclusión: la «malla de seguridad» en España (17-50). Madrid: Consejo Superior de Investigaciones Científicas.

Naik, A., Kunik, M., Cassidy, K., Nair, J. \& Coverdale, J. (2010). Assessing Safe and Independent Living in Vulnerable Older Adults: Perspectives of Professionals Who Conduct Home Assessments. JABFM- Journal of the American Board of Family Medicine, 23(5), 614-621. https://doi.org/10.3122/ jabfm.2010.05.090065

ONU. Convención de Nueva York, de 13 de diciembre de 2006, sobre los sobre los derechos de las personas con discapacidad. Instrumento de ratificación de 23 de noviembre de 2007. BOE núm. 96, de 21 abril 2008.

Palacios Rizzo, A. (2008). El modelo social de discapacidad: orígenes, caracterización y plasmación en la Convención Internacional sobre los Derechos de las Personas con Discapacidad. Madrid: CERMI.

PiZARRO, R. (2001). La vulnerabilidad social y sus desafíos: una mirada desde América Latina. Santiago de Chile: CEPAL. Disponible en http://repositorio.cepal.org/ bitstream/handle/11362/4762/S0102116_es.pdf

RAMOS PONZÓN, S. (2012). La toma de decisiones compartidas en pacientes con esquizofrenia: cuestiones médicas y éticas. Dilemata, 4 (10), s.p. Disponible en: http://www.dilemata.net/revista/index.php/dilemata/article/view/179/223

REBOURG M. \& BuRdin E. (2014), La vulnérabilité dans l'espace juridique: la situation des personnes du grand âge, en Brodiez Dolino A., Eyraud B., Laval C., Ravon B. et Von Bueltzingsloewen I. (dir.), Vulnérabilités sanitaires et sociales. Approches sociologiques et historiques, Rennes: PUR.

RendTORFF, J. D. (2002). Basic ethical principles in European bioethics and biolaw: autonomy, dignity, integrity and vulnerability. Towards a foundation of bioethics and biolaw. Medicine, Health Care and Philosophy, 5, 235-244. https://doi.org/10.1023/A:1021132602330

REQUENA MEANA, P. (2008). Dignidad y autonomía en la bioética norteamericana. Cuadernos de Bioética (66), 255-270. Disponible en: http://www.bioeticaweb. com/dignidad-y-autonomasa-en-la-bioactica-norteamericana/ 
Reino Unido. Care Act de 2014, secciones 42-7. Obtenido de http:// www.legislation.gov.uk/ukpga/2014/23/part/1/crossheading/ safeguarding-adults-at-risk-of-abuse-or-neglect/enacted.

Ricoeur, P. (2008-a). Autonomía y vulnerabilidad. En P. Ricoeur, Lo Justo II. Estudios, lecturas y ejercicios de ética aplicada (70-87). Madrid: Trotta.

RicoeUR, P. (2008-b). La diferencia entre lo normal y lo patológico como fuente de respeto. En P. Ricoeur, Lo justo II. Estudios, lecturas y ejercicios de ética aplicada (173-183). Madrid: Trotta.

RUEDA EstRADA, J.D. (2012) La incapacitación judicial y el peritaje social. Una visión desde las fundaciones tutelares. Agathos. Atención sociosanitaria y bienestar. 12 (1), 30-41

Salthouse, T. (1996). Commentary: A Cognitive Psychologist's Perspective on the Assessment of Cognitive Competency. En M. Smyer, W. K. Schaie \& M. B. Kapp. New York: Springer Publishing Company.

SCHRÖDER-ButTERFILL, E. \& MARIANTI, R. (2006). A framework for understanding old-age vulnerabilities. Ageing and Society (26), 9-35. https://doi.org/10.1017/ S0144686X05004423

SCHRÖDER-BUTTERFILL, E. (2013). Le concept de vulnérabilité et sa relation a la fragilité. En F. Béland, \& M. (. Hervé, La fragilité des personnes âgées: Définitions, controverses et perspectives d'action (205-228). Rennes. https://doi.org/10.3917/ ehesp.bela.2013.01.0203

TAYLOR, B. J. \& MCKEOWN, C. (2013). Assessing and managing risk with people with physical disabilities: the development of a safety checklist. Health, Risk \& Society, 15(2), 162-175. https://doi.org/10.1080/13698575.2013.764973

Tronto, J. C. (1998). An Ethnic of care. Generations, 22 (3), 15-20.

Tullman, D. \& Chang, B. (1999). Nursing Care of the Elderly as a Vulnerable Population. Nursing clinics of North America, 34(2), 333-334.

Wood, S. \& TANIUS, B. (2008). Impact of Dementia on Decision Making Abilities. En S. H. (Ed.), Changes in Decision-Making Capacity in Older Adults. Assesment and Intervention (91-97). New Jersey: John Wiley \& Sons, Inc.

ZsAmboK, C. \& Klein, G. (1997). Naturalistic decision making. New Yersey: Lawrence Erlbaum publishers. 